\title{
Perspective Piece \\ Case not Closed: Arguments for New Studies of the Interactions between Bed Bugs and Human Pathogens
}

\author{
Jose E. Pietri* \\ Division of Basic Biomedical Sciences, Sanford School of Medicine, University of South Dakota, Vermillion, South Dakota
}

\begin{abstract}
Bed bugs (Cimex spp.) are common ectoparasites of humans. Their ubiquity across diverse human environments combined with their blood-feeding behavior creates an ideal interface for the transmission of pathogenic microbes. Despite this potential, the current dogma is that bed bugs are not vectors of any known infectious agents. However, this conclusion is based largely on the results of studies conducted before the advent of modern molecular biology and the resurgence of bed bugs on a global scale. More importantly, a small but compelling body of modern research suggesting that bed bugs can potentially vector some human pathogens exists but is often overlooked. This article critically examines the current classification of the bed bug as an insect that does not transmit disease agents. In doing so, it highlights key knowledge gaps that still exist in understanding the potential of bed bugs as pathogen vectors and outlines several arguments for why new research on the topic is necessary.
\end{abstract}

\section{INTRODUCTION}

The tropical bed bug Cimex hempiterus (Figure 1A) and the common bed bug Cimex lectularius (Figure 1B), are bloodfeeding ectoparasitic insects that primarily feed on humans. ${ }^{1}$ Together, they have a cosmopolitan distribution that spans urban and rural environments across all six inhabited continents. These insects are considered a major nuisance pest, and their recent resurgence coupled with the ongoing development of insecticide resistance has complicated efforts to control them. ${ }^{2}$ As such, bed bugs are the subject of many modern behavioral and toxicological studies that aim to identify novel strategies to mitigate their spread. On the other hand, post-resurgence studies that examine the relationships between bed bugs and human pathogens are few and far between. Although bed bugs share many behavioral, ecological, and physiological similarities with insects that are major vectors of human pathogens, the current dogma in the field of medical entomology is that these insects do not transmit any known disease agents to humans. As a result, there has been a general lack of interest in conducting new studies of bed bug-pathogen interactions among researchers over the last decade. Some authors have gone as far as to dismiss the need to conduct additional studies on the topic, ${ }^{3,4}$ in contrast to the suggestions of government agencies, such as the U.S. CDC and U.S. Environmental Protection Agency. ${ }^{5}$

Several review articles pertaining to the involvement of bed bugs in infectious diseases have been published since the early $1960 \mathrm{~s}^{6-10}$ Together, these have described most of the various pre-resurgence laboratory experiments that have been conducted to examine the viability and replication of different pathogens inside bed bugs and a limited number of studies that have sought to conduct surveillance for human pathogens in bed bugs in nature. Because other sources of this information already exist, the intent behind this article is not to re-summarize these findings extensively. Rather, the aim of this piece was to critically address the biology of bed

*Address correspondence to Jose E. Pietri, Division of Basic Biomedical Sciences, Sanford School of Medicine, University of South Dakota, 414 East Clark St., Vermillion, SD 57069. E-mail: jose.pietri@usd.edu bugs as it pertains to their potential for disease transmission. To this end, here I present a series of arguments based largely on modern studies that contradict commonly perpetuated oversimplifications about the role of bed bugs as vectors. In doing so, I reveal that some conclusions about the ability of bed bugs to transmit pathogens may be premature and that there is still much to learn before this potential can be fully laid to rest.

Argument 1: The physiology and immunology of bed bugs do not inherently prevent them from being colonized by human pathogens. There is a lack of evidence documenting the direct transmission of any pathogen from bed bugs to humans in the primary literature. Secondary reports often conflate this fact with the idea that some innate physiological property of bed bugs must render them fundamentally unable to transmit any pathogens. ${ }^{10,11}$ However, to date, no truly viable hypothesis for why bed bugs would be biologically incapable of pathogen transmission has been put forth or investigated to an appreciable degree. The most prevalent hypotheses proposed in the literature are that infection with Wolbachia endosymbionts provides a protective effect against human pathogens, ${ }^{12}$ bed bugs encode unique antipathogen effector proteins, ${ }^{10,11,13}$ and bed bugs have evolved abnormally robust immune systems as a consequence of reproduction via traumatic insemination. ${ }^{10,11,13}$ These hypotheses still need to be explicitly tested in the context of human pathogen infection in a controlled laboratory setting, but they nonetheless appear misguided for several reasons. First, many other insects that are prolific vectors harbor natural Wolbachia infections, and the protective effect of this endosymbiont against pathogens is usually observed with transinfections. ${ }^{14}$ Similarly, microbial challenges stemming from traumatic insemination would be expected to select for immune responses that occur at the site of wounding (spermalage), which are distinct from those of the hemolymph and gut. ${ }^{15}$ Last, the genomic analysis of $C$. lectularius has revealed that antimicrobial effectors and pathogen recognition factors are underrepresented relative to holometabolous insects. ${ }^{16}$

The most compelling evidence against the aforementioned and similar hypotheses based on fundamental bed bug biology is that several species in the family Cimicidae that parasitize birds and bats are established vectors of pathogens 


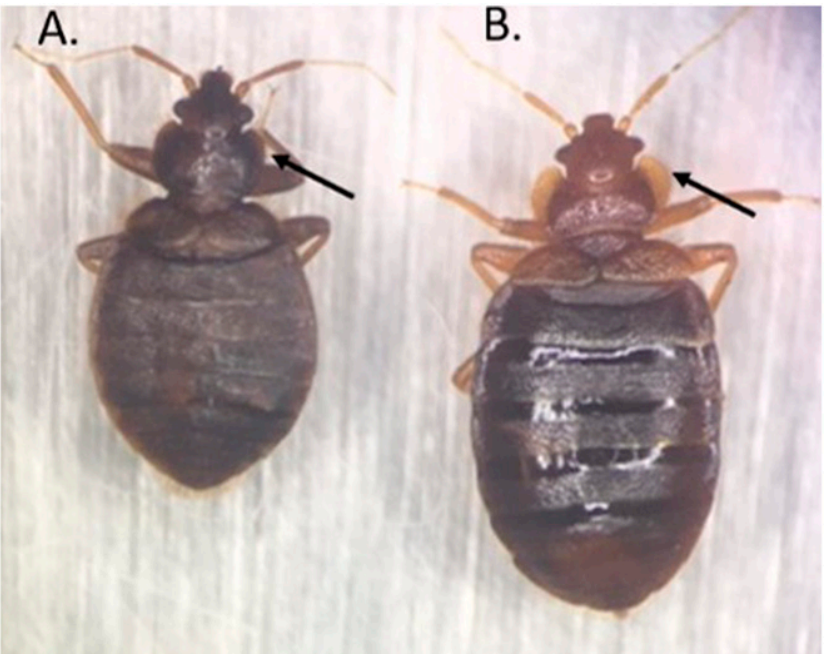

Figure 1. Bed bugs. (A) Tropical bed bug, Cimex hemipterus. (B) Common bed bug, Cimex lectularius. Photographs are of individuals from laboratory colonies. Note the difference in size and shape of the pronotum which serves as a distinguishing feature (arrows). This figure appears in color at www.ajtmh.org.

among their preferred host populations. For example, the cliff swallow bug, Oeciacus vicarious, can transmit Buggy Creek virus (Togaviridae; Alphavirus) and a second Alphavirus related to Western equine encephalitis virus, Fort Morgan virus. ${ }^{17-19}$ Similarly, there is strong evidence that the bat bugs Stricticimex parvus and Cimex adjunctus transmit the Bunyavirus Kaeng Khoi virus among free-tailed bats. ${ }^{20}$ Moreover, the bat bug Cimex brevis can transmit bat-specific trypanosomes between big brown bats. ${ }^{21}$ These closely related species of cimicid bugs, such as human bed bugs, also reproduce via traumatic insemination and can harbor Wolbachia endosymbionts. ${ }^{22,23}$ Thus, it seems unlikely that these factors would preclude human bed bugs from being colonized or shedding human pathogens.

Indeed, several recent studies have provided evidence that the natural transmission cycle of multiple human pathogenic microbes can be completed in bed bugs that were artificially infected in the laboratory. The list of these agents includes the causative agent of Chagas disease, Trypanosoma cruzi, ${ }^{24,25}$ the causative agent of trench fever, Bartonella quintana, ${ }^{26}$ and the causative agent of louse-borne relapsing fever, Borrelia recurrentis. ${ }^{27}$ An additional study suggests Rickettsia parkeri can persist in the salivary glands of bed bugs for up to 2 weeks after ingestion but did not determine infectivity. ${ }^{28}$ These modern laboratory experiments, such as pre-resurgence works on other bacterial agents, such as Yersinia pestis ${ }^{29}$ and Coxiella burnetii, ${ }^{30}$ should be confirmed through more extensive investigation. Nonetheless, considering this evidence, it is clear that, biologically speaking, bed bugs can support the development and shedding of some pathogens. Of course, this alone is not indicative of the ability to efficiently transmit the same agents in nature (i.e., vectorial capacity), but it argues against the hypothesis of a biological barrier to vector competence in bed bugs that is frequently and misleadingly put forth in the literature. Instead, it seems more likely that ecological factors could be critical drivers that may confine pathogen transmission by bed bugs to very limited settings or prevent the phenomenon in nature altogether. If this is the case, then environmental changes that spur shifts in human or bed bug populations in the future could potentially open the door for the emergence of bed bug-borne infections. Therefore, the ecological interactions between bed bugs and human pathogens should be considered more closely.

Argument 2: The behavior and ecology of bed bugs are conducive to pathogen acquisition and transmission. It is certainly a plausible hypothesis that bed bugs do not currently vector any pathogens in nature, despite being competent vectors in the laboratory because behavioral and/or ecological factors prevent sufficient contact between the insects, microbes, and human hosts, driving transmission to undetectable levels. That is, vectorial capacity can be limited by vector habitat availability, vector biting and defecation rates, vectorto-host ratio, and the extrinsic incubation period of a pathogen within a vector, all of which are influenced to some extent by environmental conditions. ${ }^{31,32}$ However, there is again some evidence to the contrary. For instance, although bed bugs can actively move between close spaces, they are also capable of passive dispersal across huge distances ${ }^{33}$ and have a habitat range that is much greater than most if not all disease vectors. Bed bugs may take a blood meal as quickly as every 3 days, but they can survive months without feeding, ${ }^{34}$ which may facilitate the transmission of pathogens with both short and long incubation periods. Similar to body lice and triatomine bugs, they defecate rapidly after feeding, allowing for the dissemination of pathogens in the feces. ${ }^{24,35}$ They are also susceptible to being crushed while engorging on a sleeping host, allowing for the dissemination of pathogens present in the hemolymph, such as lice. Finally, although they prefer to feed on humans, they feed infrequently on alternate vertebrate hosts, such as rodents ${ }^{36}$ and cats, ${ }^{37}$ which creates opportunity for the incidental acquisition of zoonotic pathogens.

In the aforementioned regards, bed bugs share similarities with several other vector species, suggesting that they too could be capable of naturally acquiring and disseminating at least some pathogens in field settings. Most louse-borne bacteria, such as $B$. quintana and $B$. recurrentis, have no nonhuman reservoir and, therefore, do not necessitate the vectors to feed on animal hosts for transmission to occur. These agents are instead transmitted from human to human by body lice in the feces and hemolymph, respectively. If bed bugs are present in dwellings where infected individuals reside, there is little reason to believe that they could not function in a role similar to lice, acquiring and disseminating these pathogens in their feces, as has been experimentally demonstrated in the laboratory. ${ }^{26,27}$ Similarly, in some limited scenarios, one can envision bed bugs acquiring Borrelia duttoni, which also has no nonhuman reservoir and is transmitted by argasid ticks in rural homes, or agents such as Rickettsia felis, Rickettsia akari, or Bartonella henselae when feeding on infected cats or rodents. However, the vector competence of bed bugs for the latter three agents has not been tested.

Although very little is known about the microbes associated with field populations of bed bugs in today's environment, limited screens have detected several human pathogens in field-collected samples, indicating that bed bugs are naturally exposed to these agents. In 2011, B. quintana was detected in a tropical bed bug from a jail in Rwanada. ${ }^{38}$ Furthermore, a tropical bed bug collected from a household in Senegal in 2012 tested positive for $R$. felis, ${ }^{39}$ a species which can colonize a diverse range of arthropods (e.g., fleas, booklice, and 
mosquitoes). In 2013, the presence of Burkholderia multivorans was reported in C. lectularius from North Carolina. ${ }^{40}$ Several samples more recently collected from the United States and United Kingdom were also shown to harbor an uncharacterized ancestral Rickettsia. ${ }^{41}$ Even if naturally exposed to pathogens for which they are competent vectors, some biological parameters may limit the transmission capacity of bed bugs. For instance, bed bugs may require higher pathogen doses than other insects to become stably infected. This possibility has been suggested to explain why no cases of T. cruzi transmission by bed bugs have been documented. ${ }^{42}$ Alternatively, because of differences in erythrolysis kinetics during the blood digestion process, the rate of pathogen shedding by bed bugs may be too low to naturally infect humans to any appreciable degree. ${ }^{43}$ However, neither of these hypotheses has been investigated experimentally. Given the ubiquity of bed bugs across human environments and recent preliminary observations of natural exposures to pathogens, there remains a critical need to conduct wider surveys of pathogen prevalence in field populations. These are currently nonexistent beyond a few geographically restricted investigations that targeted a narrow range of bacterial pathogens. ${ }^{40,41,44}$ Such studies should especially focus on microbes for which vector competence has already been confirmed (see the previous paragraphs) and will go a long way toward disproving or establishing the transmission of these agents, as the prevalence of infected vectors is an important factor in the determination of vectorial capacity. Moreover, virome studies to identify novel, potentially pathogenic viruses in bed bugs should also be of interest. Alphaviruses and bunyaviruses are particularly intriguing candidates, given their prevalence in other cimicids. ${ }^{8,17-20}$

Argument 3: Studies to date have been inadequate to disprove the hypothesis that bed bugs may vector certain agents of disease in nature. Perhaps, the most pervasive argument against the potential of bed bugs as vectors in nature is that if bed bugs were transmitting disease to humans, this phenomenon would have already been observed. This argument is not only an appeal to ignorance fallacy, but it is also highly unscientific, relying only on a lack of evidence and discounting the stochastic nature of pathogen emergence. Although, of course, it is impossible to prove a negative, a collection of negative evidence can provide strong support for a negative argument. Yet, the magnitude of the knowledge gap that exists in understanding the interactions between bed bugs and pathogens is often severely understated.

Almost all studies on the topic were conducted before the advent of modern molecular biology and well before the bed bug resurgence. Mostly, this literature consists of laboratorybased studies that often lack rigor (e.g., proper controls, replication, and clear descriptions of methods and results) and include a significant amount of conjecture and anecdotes. Even so, vector competence for many agents, including Rickettsia typhi, ${ }^{45}$ yellow fever virus, ${ }^{46}$ filarial worms, ${ }^{47}$ and several others, has been ruled out by negative data. ${ }^{6-10} \mathrm{Al}-$ though the reality is that bed bugs would not be expected to vector such agents given what we now know about their biology, there are many others for which transmission appears much more plausible based on current evidence (e.g., Bartonella quintana, $B$. recurrentis, and $R$. felis). The greatest barrier to understanding the true capacity for bed bugs to transmit these agents is simply a lack of relevant studies. For example, although $R$. felis was detected in bed bugs in the field several years ago and is known to be a cause of febrile illness of unknown origin across Africa, no vector competence studies of this agent have been reported. Similarly, vector competence for $B$. henselae has not been tested, despite evidence that bed bugs are competent vectors of the closely related bacterium $B$. quintana. Conversely, minimal attempts have been made to conduct surveillance for bacterial pathogens that bed bugs are competent vectors in populations of the insects that are present where these agents circulate, such as homeless shelters and camps, or homes in developing nations. ${ }^{48-50}$ More critically, clinical studies involving humans exposed to bed bugs that could potentially reveal associations with known or novel infectious diseases are completely lacking. Similar studies have revealed cryptic roles for mesostigmatid mites as possible vectors of Bartonella spp. ${ }^{51,52}$ In sum, there is not nearly as much firm negative evidence against pathogen transmission by bed bugs as some reports may suggest.

Although one may make a fair case that if bed bugs were causing major disease epidemics this would likely have been observed at some point, a role for the bed bug as a secondary vector of a known disease agent or as a vector of a rare or novel pathogen with a low disease burden is much more difficult to rule out. In such situations, disease cases caused by bed bugs could be misattributed to other sources such as primary vectors or go undetected without thorough investigation. The relationship between $R$. akari, the causative agent of rickettsialpox, and its vector the house mouse mite, Liponyssoides sanguineus, provides an intriguing example. ${ }^{53}$ Rickettsialpox is a relatively mild, self-limiting febrile illness and no deaths from the infection have been reported. The disease was first discovered in New York City in 1946 after a string of infections in a housing development prompted a team of physicians, epidemiologists, and entomologists to investigate in real time. These inquiries soon led to the isolation of the causative agent from afflicted humans as well as mice and mites in the building, establishing the zoonotic cycle. Since this time, $\sim 1,000$ cases of rickettsialpox have been reported worldwide. Most of these have been from New York City and have simply been assumed to be the result of mite bites without direct evidence. Barring the opportune 1946 investigation, it is quite possible that the transmission cycle of rickettsialpox could have remained undocumented for much longer. Because of the rarity of the infection, its mild symptoms, and the elusiveness of the mite vector, the disease is thought to be underreported and direct transmission is almost never documented. Yet, it would be naive to think that $R$. akari did not circulate before its discovery and does not continue to circulate today. Although it may be difficult to imagine bed bugs triggering major outbreaks of vector-borne disease, it is much less difficult to imagine a vector role for the bed bug that is similar in nature to that of the house mouse mite, occurring at limited points in space and time. Such a role would be no less scientifically interesting, transformative, and important to identify from a public health perspective.

Louse-borne bacteria as candidate bed bug-borne pathogens in today's environment. Among the various human pathogens that have been investigated in bed bugs, there is in fact one agent for which nearly all criteria necessary to classify the bed bug as a vector have at least some support: B. quintana, which primarily afflicts marginalized populations, 
such as the homeless, causing trench fever, chronic bacteremia, endocarditis, and bacillary angiomatosis. ${ }^{54}$ Because of its restriction to marginalized populations, it is considered by many to be a "silent epidemic." The established vector of $B$. quintana is the human body louse, which enables substantial bacterial replication and excretes the pathogen in its feces. In bed bugs, replication of $B$. quintana appears limited, but the bacteria are able to survive and are shed in the feces in viable form for at least 3 days under laboratory conditions, fulfilling key biological prerequisites for transmission. ${ }^{26}$ More importantly, both bed bugs and body lice are known to thrive in environments of high population density and low hygiene, such as homeless shelters, suggesting an ecological overlap between the two insects. ${ }^{55}$ Indeed, co-infestation with both ectoparasites was recently documented in an individual from the United States. ${ }^{56}$ Similarly, in jails in Rwanda, outbreaks of louse-borne trench fever are common. ${ }^{57}$ It is in this setting that $B$. quintana was first detected in the tropical bed bug in 2011. ${ }^{37}$ Angelakis et al., ${ }^{38}$ thus, represented an important landmark, being the first post-resurgence study to achieve field detection of a pathogen for which there is also evidence that bed bugs are competent vectors. Unfortunately, the authors of that work were unable to collect samples from humans or lice at the same location and, therefore, could not establish the role of bed bugs in transmission. Currently, the documentation of infected bed bugs harboring viable $B$. quintana in an epidemic setting remains a missing piece of the puzzle, but given existing evidence, it appears possible that bed bugs may eventually be recognized as vectors. The relationship between $B$. quintana and bed bugs again exemplifies the complexities behind establishing a novel vector for a pathogen with a restricted disease burden. That is, because $B$. quintana circulates primarily among medically neglected populations and often goes undiagnosed, studies to rigorously examine the contribution of bed bugs to transmission in the field will require careful coordination between a combination of social workers, clinicians, and laboratory scientists to obtain samples from insects and patients at the right place and time. In Figure 2, I propose several plausible scenarios in which bed bugs could contribute to the transmission of $B$. quintana that should be considered in future clinical and epidemiological studies. Among the factors that remain to be determined are whether the titers of $B$. quintana that occur naturally in human infections are sufficient to infect bed bugs for any appreciable period of time, and whether the rates of bacterial shedding and bed bug feeding on naive human hosts are sufficient to drive transmission. Notably, the scenarios proposed in Figure 2 are generically applicable to other louseborne pathogens for which humans are the reservoir and that spread under crowded, low-hygiene conditions, such as $B$. recurrentis, which bed bugs may also be competent vectors of. 27

\section{CONCLUDING REMARKS AND FUTURE DIRECTIONS IN BED BUG-PATHOGEN RESEARCH}

Here, I have outlined several arguments that support the potential of bed bugs as pathogen vectors. Based on existing evidence, it is not warranted to classify bed bugs as vectors at this time. However, given the biology and prevalence of these insects, it would be irresponsible to dismiss this possibility and future studies that seek to address it. Much of the preresurgence literature on bed bug-pathogen interactions is poorly described, lacking in scientific rigor, or focused on pathogens for which no vector-borne cycle is known (e.g., HIV and hepatitis $B$ virus). ${ }^{58,59}$ On the other hand, modern studies have been limited in number and scope. In particular, although bed bugs have already been found to be competent vectors of several louse-borne bacteria in preliminary laboratory tests, detailed studies of the kinetics of these infections are

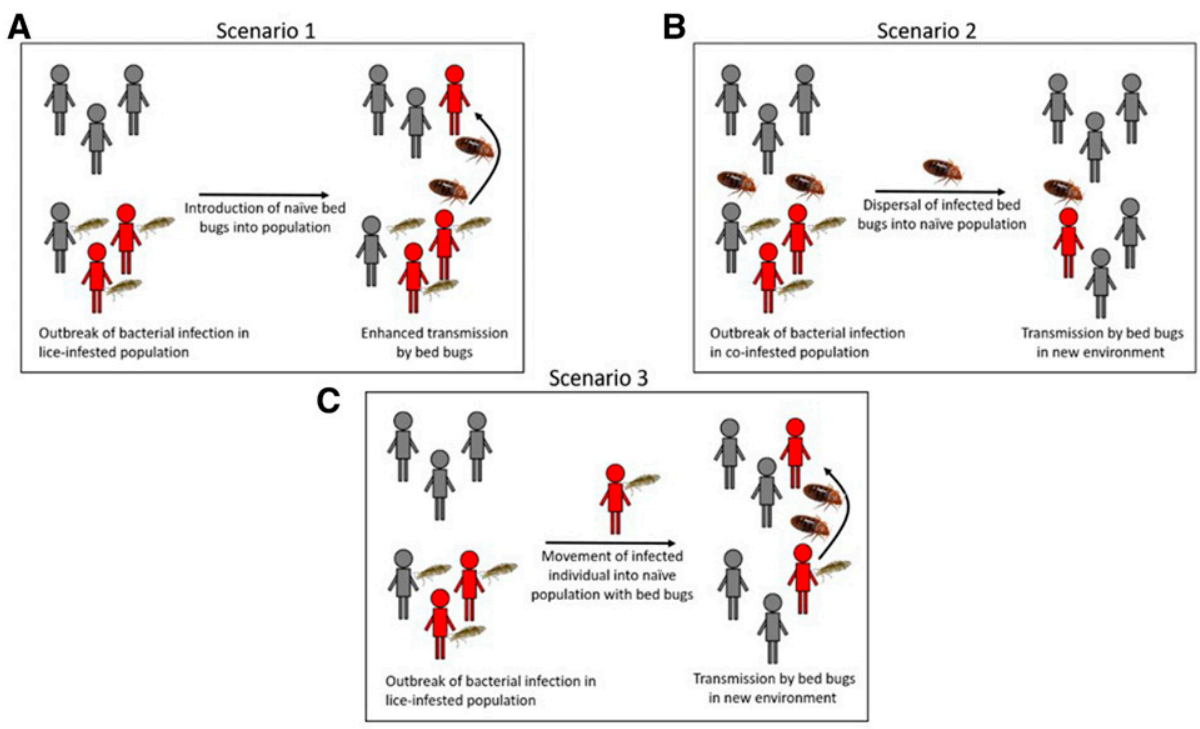

FIGURE 2. Hypothetical scenarios enabling secondary transmission of louse-borne bacteria by bed bugs. The ecological overlap between body lice and bed bugs in select environments presents several plausible opportunities for bed bugs to contribute to transmission of several bacterial pathogens. Bed bugs could (A) enhance transmission when introduced into a population with existing louse-borne pathogen transmission, (B) facilitate transmission from a co-infested population to a naive population via dispersal, or (C) facilitate transmission from an infected individual migrating into a bed bug-infested population. These potential avenues should be explored more closely through future studies. This figure appears in color at www.ajtmh.org. 
necessary to better understand how vector competence may or may not translate to natural scenarios. Similarly, the use of rapidly developing high-throughput DNA and RNA sequencing to carry out geographically diverse pathogen surveillance and discovery studies of bed bugs could reveal associations with additional and/or novel pathogens and help us to understand infection prevalence. Meanwhile, clinical studies involving bed bug-infested human populations will be critical to answering the ultimate question of whether or not bed bugs can directly transmit any agents that lead to disease. More broadly, the conundrum surrounding the role of bed bugs as vectors underscores the importance of negative data in reaching scientific consensus. In addressing this phenomenon, the publication of negative results demonstrating a lack of vector competence or lack of pathogen detection in relevant field samples will be essential to ruling out transmission of pathogens of interest by bed bugs and should be encouraged. Until more of these studies are publicly disseminated or cases of human disease that can be directly attributed to bed bugs are identified, speculation and some controversy will inevitably continue.

Received February 12, 2020. Accepted for publication March 19, 2020.

Published online April 27, 2020.

Author's address: Jose E. Pietri, Division of Basic Biomedical Sciences, Sanford School of Medicine, University of South Dakota, SD, E-mail: jose.pietri@usd.edu

\section{REFERENCES}

1. Williams KW, Willis MS, 2012. Bed bugs in the 21st century: the reemergence of an old foe. Lab Med 43: 141-148.

2. Dang K, Doggett SL, Singham GV, Lee CW, 2017. Insecticide resistance and resistance mechanisms in bed bugs Cimex spp. (Hemiptera: Cimicidae). Parasit Vectors 10: 308.

3. Doggett SL, 2018. Chapter 12: bed bugs and infectious diseases. Advances in the Biology and Management of Modern Bed Bugs. Hoboken, NJ: Wiley-Blackwell, 117-122.

4. Doggett SL, Dwyer DE, Penas PF, Russell RC, 2012. Bed bugs: clinical relevance and control options. Clin Microbiol Rev 25: 164-192.

5. US Centers for Disease Control and Prevention and US Environmental Protection Agency, 2010. Joint Statement on Bed Bug Control in the United States. Atlanta, GA: US Department of Health and Human Services.

6. Burton GJ, 1963. Bed bugs in relation to transmission of human diseases. Public Health Rep 78: 513-524.

7. Delaunay P, Blanc V, Del Giudice P, Levy-Bencheton A, Chosidow $O$, Marty $P$, Brouqui $P, 2011$. Bedbugs and infectious diseases. Clin Infect Dis 52: 200-210.

8. Adelman ZN, Miller DM, Myles KM, 2012. Bed bugs and infectious disease: a case for the arboviruses. PLoS Pathog 9: e1003462.

9. Zorrila-Vaca A, Silva-Medina MM, Escandon-Vargas K, 2015. Bedbugs, Cimex spp.: their current world resurgence and healthcare impact. Asian Pac J Trop Dis 5: 342-352.

10. Lai O, Ho D, Glick S, Jagdeo J, 2016. Bed bugs and possible transmission of human pathogens: a systematic review. Arch Dermatol Res 308: 531-538.

11. Ho D, Lai O, Glick S, Jagdeo J, 2016. Lack of evidence that bedbugs transmit pathogens to humans. J Am Acad Dermatol 74: 1261.

12. Fisher ML, Levine JF, Guy JS, Mochizuki H, Breen M, Schal C, Watson DM, 2019. Lack of influence by endosymbiont Wolbachia on virus titer in the common bed bug, Cimex lectularius. Parasit Vectors 12: 436.

13. Cockburn $C$ et al., 2013. Gram-positive bacteria isolated from the common bed bug, Cimex lectularius L. Entomol Am 119: 23-29.
14. Lindsey ARI, Bhattacharya T, Newton ILG, Hardy RW, 2018. Conflict in the intracellular lives of endosymbionts and viruses: a mechanistic look at Wolbachia-mediated pathogen blocking. Viruses 10: 141

15. Morrow EH, Arnqvist G, 2003. Costly traumatic insemination and a female counter-adaptation in bed bugs. Proc $R$ Soc Lond $B$ 270: 2377-2381.

16. Benoit JB et al., 2016. Unique features of a global human ectoparasite identified through sequencing of the bed bug genome. Nat Commun 7: 10165.

17. Brown CR, Moore AT, Young GR, Komar N, 2010. Persistence of buggy creek virus (Togaviridae, Alphavirus) for two years in unfed swallow bugs (Hemiptera: Cimicidae: Oeciacus vicarious). J Med Entomol 47: 436-441.

18. Hayes RO, Francy DB, Lazuick JS, Smith GC, Gibbs PJ, 1977. Role of the cliff swallow bug (Oeciacus vicarious) in the natural cycle of a western equine encephalitis-related Alphavirus. J Med Entomol 14: 257-262.

19. Rush WA, Francy DB, Smith GC, Cropp CB, 1980. Transmission of an arbovirus by a member of the family Cimicidae. Ann Entomol Soc Am 73: 315-318.

20. Williams JE, Imlarp S, Top FH, Cavanaugh DC, Russell PK, 1976. Kaeng Khoi virus from naturally infected bedbugs (Cimicidae) and immature free-tailed bats. Bull World Health Organ 53: 365-369.

21. Bower SM, Woo PTK, 1981. Development of trypanosoma (Schizotrypanum) hedricki in Cimex brevis (Hemiptera: Cimicidae). Can J Zool 59: 546-554.

22. Rasgon JL, Scott TW, 2004. Phylogenetic characterization of Wolbachia endosymbionts infecting Cimex lectularius L. and Oeciacus vicarius Horvath (Hemiptera: Cimicidae). J Med Entomol 41: 1175-1178.

23. Sakamoto JM, Feinstein J, Rasgon JL, 2006. Wolbachia infections in the Cimicidae: museum specimens as an untapped resource for endosymbiont surveys. Appl Environ Microbiol 75: 3161-3167.

24. Salazar R, Castillo-Neyra R, Tustin AW, Borrini-Mayori K, Naquira C, Levy MZ, 2015. Bed bugs (Cimex lectularius) as vectors of Trypanosoma cruzii. Am J Trop Med Hyg 92: 331-335.

25. Blakely BN, Hanson SF, Romero A, 2018. Survival and transstadial persistence of Trypanosoma cruzii in the bed bug (Hemiptera: cimicidae). J Med Entomol 55: 742-746.

26. Leulmi H, Bitam I, Berenger JM, Lepidi H, Rolain JM, Almeras L, Raoult D, Parola P, 2015. Competence of Cimex lectularius bed bugs for the tranmission of Bartonella quintana, the agent of trench fever. PLoS Negl Trop Dis 9: e0003789.

27. El Hamzaoui B, Laroche M, Bechah Y, Berenger JM, Parola P, 2019. Testing the competence of Cimex lectularius bed bugs for the transmission of Borrelia recurrentis, the agent of relapsing fever. Am J Trop Med Hyg 100: 1407-1412.

28. Goddard J, Varela-Stokes A, Smith W, Edwards KT, 2012. Artificial infection of the bed bug with Rickettsia parkeri. J Med Entomol 49: 922-926.

29. Bacot AW, 1915. Notes on the development of Bacillus pestis in bugs (Cimex lectularius) and their power to convey infection. J Hyg (Lond) 14: 777-792.

30. Weyer $F, 1962$. Observations on significance of bedbugs as carriers of Rickettsia. Z Für Angew Zool 49: 61-78.

31. Magori K, Drake JM, 2013. The population dynamics of vectorborne diseases. Nat Educ 4: 14.

32. LaDeau SL, Allan BF, Leisnham PT, Levy MZ, 2016. The ecological foundations of transmission potential and vector-borne disease in urban landscapes. Funct Ecol 29: 889-901.

33. Hentley WT, Webster B, Evison SEF, Siva-Jothy MT, 2017. Bed bug aggregation on dirty laundry: a mechanism for passive dispersal. Sci Rep 7: 11668.

34. Reinhardt K, Isaac D, Naylor R, 2010. Estimating the feeding rate of the bed bug Cimex lectularius in an infested room: an inexpensive method and a case study. Med Vet Entomol 24: 46-54.

35. Darrington CL, 2015. Bed Bug (Cimex lectularius) Defecation Behavior Following a Bloodmeal. Dissertation, The Ohio State University.

36. Rivnay E, 1930. Host selection and cannibalism in the bed bug Cimex lectularius L. Ann Entomol Soc Am 23: 758-764. 
37. Clark S, Gilleard JS, McGoldrick J, 2002. Human bedbug infestation of a domestic cat. Vet Rec 151: 336.

38. Angelakis E, Socolovschi C, Raoult D, 2013. Bartonella quintana in Cimex hemipterus, Rwanda. Am J Trop Med Hyg 89: 986-987.

39. Mediannikov O et al., 2013. Common epidemiology of Rickettsia felis infection and malaria, Africa. Emerge Infect Dis 19: 1775-1783.

40. Saenz VL, Maggi RG, Breitschwerdt EB, Kim J, Vargo EL, Schal C, 2013. Survey of Bartonella spp in U.S. bed bugs detects Burkholderia but not Bartonella. PLoS One 8: e73661.

41. Potts RA, Molina I, Sheele JM, Pietri JE, 2020. Molecular detection of Rickettsia infection in field-collected bed bugs. New Microbes New Infect 34: 100646.

42. Peterson JK, Salazar R, Castillo-Neyra R, Borrini-Mayori K, Condori C, Bartow-McKenney C, Tracy D, Naquira C, Levy MZ, 2018. Trypanosoma cruzii infection does not decrease survival or reproduction of the common bed bug, Cimex lectularius. Am J Trop Med Hyg 98: 724-734.

43. Vaughan JA, Azad AF, 1993. Patterns of erythrocyte digestion by bloodsucking insects: contraints on vector competence. J Med Entomol 30: 214-216.

44. Richard S, Peng P, Parola P, Raoult D, Davoust B, Brouqui P, 2009. Detection of a new bacterium related to 'Candidatus Midichloria mitochondrii' in bed bugs. Clin Microbiol Infect 15: 84-85.

45. Nauck EG, Zumpt F, 1940. The question of the transmission of murine typhus by the bed bug. Zentralbl fur Bakteriol Parasitenkd Infekt Hyg Abt I (Orig) 146: 97-103.

46. Kumm HW, Frobisher M, 1932. Attempts to transmit yellow fever with certain Brazilian mosquitoes (Culicidae) and with bedbugs (Cimex hemipterus). Am J Trop Med Hyg s1-12: 349-361.

47. Wharton $\mathrm{RH}$, Omar $\mathrm{AH}, 1962$. Failure of Wuchereria bancrofti and Brugia malayi to develop in the tropical bed bug Cimex hemipterus. Ann Trop Med Parasitol 56: 188-190.

48. Brouqui $P$, Raoult $D, 2006$. Arthropod-borne diseases in homeless. Ann NY Acad Sci 1078: 223-235.
49. Brouqui $P$, Stein A, Dupont HT, Gallian P, Badiaga S, Rolain JM, Mege JL, La Scola B, Berbis P, Raoult D, 2005. Ectoparasitism and vector-borne diseases in 930 homeless people from Marseilles. Medicine (Baltimore) 84: 61-68.

50. Leibler JH, Robb K, Joh E, Gaeta JM, Rosenbaum M, 2018. Selfreported animal and ectoparasite exposure among urban homeless people. J Health Care Poor Underserved 29: 664-675.

51. Bradley JM, Mascarelli PE, Trull CL, Maggi RG, Breitschwerdt EB, 2014. Bartonella henselae infections in an owner and two $\mathrm{Pa}$ pillon dogs exposed to tropical rat mite (Ornithonyssus bacoti). Vector Borne Zoonotic Dis 14: 703-709.

52. Melter O, Arvand M, Votypka J, Hulinska D, 2012. Bartonella quintana transmission from mite to family with high socioeconomic status. Emerge Infect Dis 18: 163-165.

53. Paddock CD, Zaki SR, Koss T, Singleton J, Summer JW, Comer JA, Eremeeva E, Dasch GA, Cherry B, Childs JE, 2003. Rickettsialpox in New York city: a persistent urban zoonosis. Ann NY Acad Sci 990: 36-44.

54. Cheslock MA, Embers ME, 2019. Human bartonellosis: an underappreciated public health problem? Trop Med Infect Dis 4: 69.

55. Hwang SW, Svodova TJ, DeJong I, Kabasele KJ, Gogosis E, 2005. Bed bug infestations in an urban environment. Emerg Infect Dis 11: 533-538.

56. Pietri JE, Yax JA, Agany DDM, Gnimpieba EZ, Sheele JM, 2020. A case of body lice and bed bug co-infestation in an emergency department patient, Ohio, USA. IDCases 19: e00696.

57. Fournier PE, Ndihokubwayo JB, Guidran J, Kelly PJ, Raoult D, 2008. Human pathogens in body and head lice. Emerg Infect Dis 8: 1515-1518.

58. Blow JA, Turell MJ, Silverman AL, Walker ED, 2001. Stercorarial shedding and transtadial transmission of hepatitis $B$ virus by common bed bugs (Hemiptera: Cimicidae). J Med Entomol 38: 694-700.

59. Lyons SF, Jupp PG, Schoub BD, 1986. Survival of HIV in the common bed bug. Lancet 2: 45 . 